\title{
Phase transitions and steady-state microstructures in a two-temperature lattice-gas model with mobile active impurities
}

Henriksen, Jonas Rosager; Sabra, Mads Christian; Mouritsen, Ole G.

Published in:

Physical Review E. Statistical, Nonlinear, and Soft Matter Physics

Link to article, DOI:

10.1103/PhysRevE.62.7070

Publication date:

2000

Document Version

Publisher's PDF, also known as Version of record

Link back to DTU Orbit

Citation (APA):

Henriksen, J. R., Sabra, M. C., \& Mouritsen, O. G. (2000). Phase transitions and steady-state microstructures in a two-temperature lattice-gas model with mobile active impurities. Physical Review E. Statistical, Nonlinear, and Soft Matter Physics, 62(5), 7070-7076. https://doi.org/10.1103/PhysRevE.62.7070

\section{General rights}

Copyright and moral rights for the publications made accessible in the public portal are retained by the authors and/or other copyright owners and it is a condition of accessing publications that users recognise and abide by the legal requirements associated with these rights.

- Users may download and print one copy of any publication from the public portal for the purpose of private study or research.

- You may not further distribute the material or use it for any profit-making activity or commercial gain

- You may freely distribute the URL identifying the publication in the public portal 


\title{
Phase transitions and steady-state microstructures in a two-temperature lattice-gas model with mobile active impurities
}

\author{
Jonas R. Henriksen, Mads C. Sabra, and Ole G. Mouritsen* \\ MEMPHYS, Department of Chemistry, Technical University of Denmark, Building 206, DK-2800 Lyngby, Denmark
}

(Received 7 April 2000)

\begin{abstract}
The nonequilibrium, steady-state phase transitions and the structure of the different phases of a twodimensional system with two thermodynamic temperatures are studied via a simple lattice-gas model with mobile active impurities ("hot/cold spots") whose activity is controlled by an external drive. The properties of the model are calculated by Monte Carlo computer-simulation techniques. The two temperatures and the external drive on the system lead to a rich phase diagram including regions of microstructured phases in addition to macroscopically ordered (phase-separated) and disordered phases. Depending on the temperatures, microstructured phases of both lamellar and droplet symmetry arise, described by a length scale that is determined by the characteristic temperature controlling the diffusive motion of the active impurities.

PACS number(s): 87.17.-d, 05.70.Ln, 64.60.Cn, 64.75.+g
\end{abstract}

\section{INTRODUCTION}

The structuring of most solids on the scale from molecules or atoms over the mesoscopic range to macroscopic length scales, is predominantly determined by forces of enthalpic origin. In contrast, for liquid mixtures or complex fluids, entropic forces often play a crucial role both for the macroscopic state of the system as well as its structure on small scales. In particular, soft and fluctuating interfaces are seminal for determining the stability of systems in this class of materials [1]. Specific examples include microemulsions [2] and microphase separated lamellar structures such as those found in block copolymer systems [3].

Due to sluggish dynamics or kinetic barriers, the microstructure of liquid mixtures, in particular those involving polymers and surfactants, can be a consequence of nonequilibrium conditions. Under ideal conditions, microstructural organization can also arise in the case of a mixture of two immiscible liquids that tend to phase separate but where the phase-separation process is impeded or has come to a halt due to the presence of impurities which couple to the dynamics of the interfacial network that is formed during the phaseseparation process [4]. This will then lead to a microstructure characterized by a length scale that is inversely proportional to the impurity concentration. Depending on the composition, this structure can be of the droplet type or bicontinuous, sometimes with a lamellar appearance. In the case where the impurities are interfacially active, e.g., surfactants, these will be enriched in the interfaces between the microphases. The range of thermodynamic stability of these phases can be very difficult to assess.

A possibility exists for controlling the range of stability of microstructured liquid mixtures and the length scale of the structure by manipulating the mixture into a nonequilibrium state that is controlled by a steady-state condition. This possibility was recently examined within a simple lattice-gas

\footnotetext{
*Author to whom correspondence should be addressed. FAX: +45-45-934808. Email address: ogm@kemi.dtu.dk
}

model with mobile active impurities [5] and proposed as a model of organization and compartmentalization of lipid membranes associated with active proteins [6]. Within this model, the active impurities couple to the interface between regions enriched in one of the two immiscible species of the binary mixture, simply by changing in a two-state manner their relative affinity to the two species. The activity of the impurities is controlled by an external drive $\Gamma$, which controls the probability of changing the affinity of the impurity, as well as a temperature $T_{2}$, which is only sensed by the impurities. This temperature can be different from the temperature $T_{1}$ of the bath to which the two major species of the mixtures are coupled and that controls the diffusive motion and interdiffusion of the two species. Formally, the model is therefore a two-temperature model in the language of drivendiffusive systems [7]. The model is related to models where a chemical reaction occurring at the interface can lead to a steady-state microstructuring of a binary system [8-16].

In our previous work, we studied the limiting case of $T_{2}$ $\rightarrow \infty$ for various values of the driving strength $\Gamma$ and showed how the length scale of the microstructured states was controlled by $\Gamma$ via an algebraic relation $[5,6]$. In the present paper we shall, for fixed $\Gamma$, describe the full steady-state phase behavior of the model in the plane spanned by the two temperatures, the diffusion temperature $T_{1}$ and the impurity temperature $T_{2}$. In the case where $T_{1} \neq T_{2}$, the impurities may be perceived as hot or cold spots where energy flows through the system from one heat bath to the other. The corresponding dissipation of energy leads to a microstructuring of the system. Due to the fact that the hot/cold spots are localized and mobile throughout the system, this flow of energy does not lead to global transport of heat as is the case for nonequilibrium systems with a global thermal gradient [17]. Other lattice-gas models with two temperatures have been considered [18-27]. In these models, the two temperatures either correspond to different diffusive temperatures for exchange of particles in two different directions on the lattice or to different temperatures on two fixed interpenetrating sublattices. In both these cases, the steady-state phases have the same symmetry as those of the equilibrium system and 

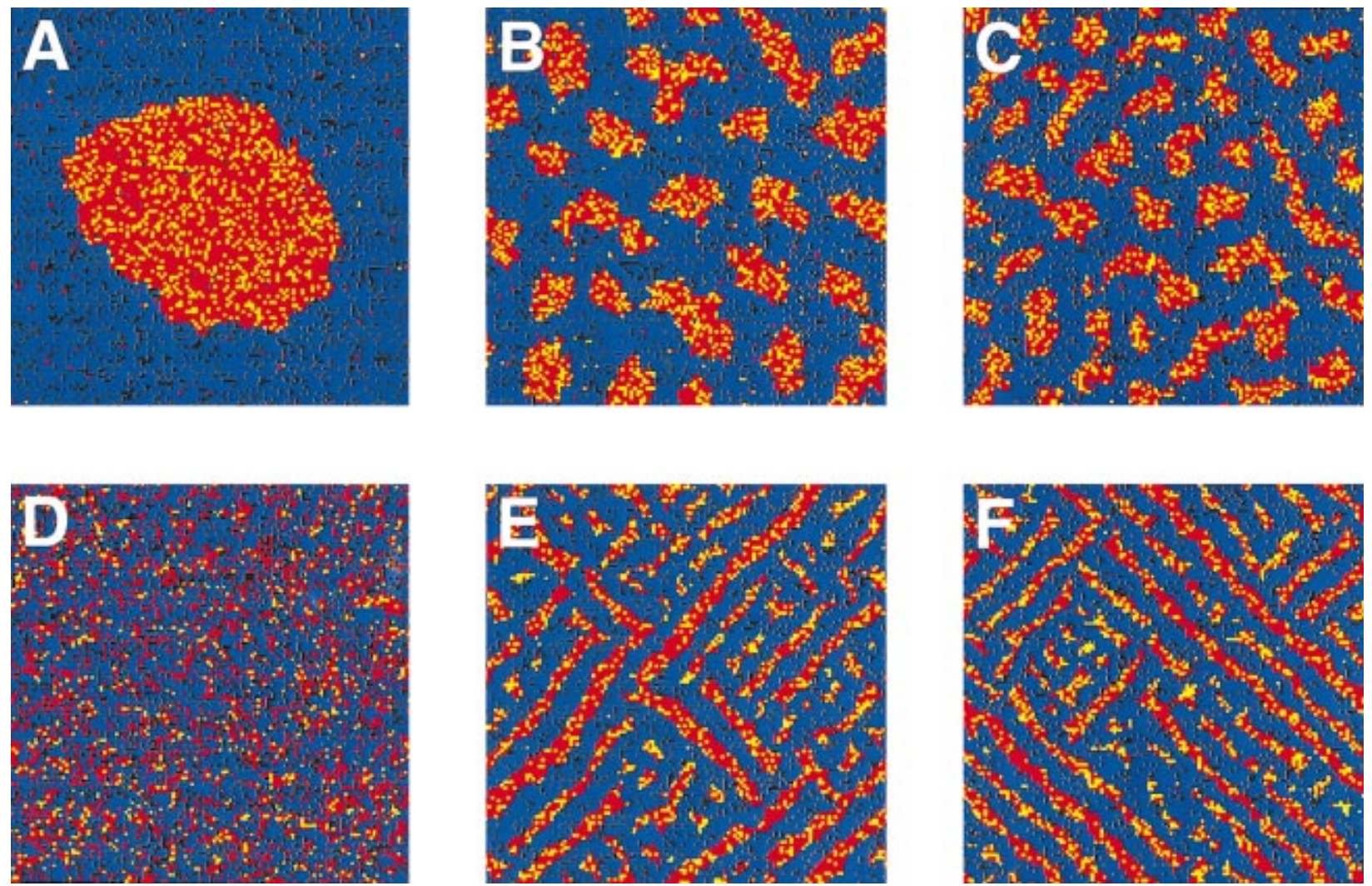

FIG. 1. (Color) Computer simulation snapshots of characteristic microconfigurations in the steady-state limit of a binary lattice-gas model with active impurities described by two temperatures, the diffusive temperature $T_{1}$, and the impurity temperature $T_{2}$. The level of impurity activity is $\Gamma=10^{-3}$. The snapshots shown correspond to different points in the phase diagram (cf. Fig. 2$),(\mathrm{A})\left(T_{1}, T_{2}\right)=(1.5,1.5) ;(\mathrm{B})$ $\left(T_{1}, T_{2}\right)=(1.5,5) ;(\mathrm{C})\left(T_{1}, T_{2}\right)=(1.5,10) ;(\mathrm{D})\left(T_{1}, T_{2}\right)=(4,1.5) ;(\mathrm{E})\left(T_{1}, T_{2}\right)=(0.5,10)$; and $(\mathrm{F})\left(T_{1}, T_{2}\right)=(0.5,100)$. All temperatures are in units of $J / \mathrm{k}_{\mathrm{B}}$. The composition of the binary mixture is $7: 3$ with $20 \%$ impurities. The system size is $128 \times 128$. The two spin species are shown in blue and red, and the impurities in the corresponding spin states are shown in black and yellow, respectively.

there is no nonequilibrium mechanism for breaking the symmetry and forming microstructured phases. It is the particular combination of two temperatures with two diffusive subsets of particles that opens up the possibility of the type of microstructuring described in the present paper.

In Sec. II, we define in detail the model studied and describe the simulation methods used to determine its properties in the steady state. The results for the different phases and phase transitions, including the steady-state phase diagram, are presented in Sec. III. The paper is concluded and summarized in Sec. IV.

\section{MODEL AND COMPUTATIONAL TECHNIQUES}

\section{A. Model}

The lattice-gas model studied in this paper is most conveniently defined within the Ising formalism by the spin- $1 / 2$ Hamiltonian

$$
\mathcal{H}=-\frac{J}{2} \sum_{i \in \Omega_{\mathrm{L}}, \Omega_{\mathrm{P}}} \sum_{j}^{\mathrm{nn}} \sigma_{i} \sigma_{j}
$$

where $\sigma_{i}= \pm 1$. The second sum is restricted to nearest neighbors of site $i$ on a square lattice with $N$ sites and periodic boundary conditions. The enumeration of the lattice sites is divided into two sets of indices, $\Omega_{\mathrm{L}}$ and $\Omega_{\mathrm{P}}$, where $\Omega_{\mathrm{L}}$ refers to the two major species. The actual type of the species on a given site $i$ is determined by the sign of $\sigma_{i}$. Similarly, $\Omega_{\mathrm{P}}$ labels the impurities, and the internal states of the impurities are given by the corresponding values of $\sigma_{i}$.

All interactions in Eq. (1) are scaled by the same positive interaction constant $J$, which corresponds to ferromagnetic (attractive) interactions. By this choice, an impurity has affinity for the type of spin species to which its spin state corresponds. In ordinary thermal equilibrium, Eq. (1) therefore describes exactly the same thermodynamic state as the conventional spin-1/2 Ising. However, in the present description the model contains a fixed and identifiable set of the spins, called the impurities, which are labeled to be prone for activity if a suitable dynamics is imposed on the model. It is only when the activity is turned on that the model is different from a conserved spin-1/2 Ising ferromagnet. In contrast to many other studies of dilute and impure Ising models, the impurities in the present model are not spin-zero particles. Instead they have the same spin states as the other spins; only their dynamics is different as described below.

The special nonequilibrium properties of the model in Eq. (1) are controlled by the following dynamics.

(i) The concentrations of the two major species and the impurities are taken to be conserved.

(ii) The diffusive dynamics of the system is controlled by nearest-neighbor Kawasaki particle exchange between any particle pair (spins or impurities). The acceptance criterion for an exchange is given by the Monte Carlo Metropolis rate, 
$\min \left\{1, \exp \left(-\Delta \mathcal{H} / \mathrm{k}_{\mathrm{B}} T_{1}\right)\right\}$, involving the diffusion temperature $T_{1}$. All pair exchanges are for simplicity taken to occur on the same time scale $\tau_{0}$, where $\tau_{0}=1$ in units of Monte Carlo steps per lattice site (MCS), i.e., the two major species and the impurities have the same diffusion constant.

(iii) The impurities are made active by subjecting them at random to an internal transition $\sigma_{i} \rightarrow-\sigma_{i}$, using Glauber spin-flip dynamics that does not conserve the magnetization. This internal change, which can be imagined to take place due to an external drive, occurs at a time scale $\tau$ (in units of $\left.\tau_{0}\right)$. The strength of the drive can therefore conveniently be described by a parameter $\Gamma=\tau^{-1}$. The acceptance criterion for changing the state of the impurity is the Monte Carlo Metropolis criterion, $\min \left\{1, \exp \left(-\Delta \mathcal{H} / \mathrm{k}_{\mathrm{B}} T_{2}\right)\right\}$, involving the impurity temperature $T_{2}$.

According to this dynamics, the relation between the actual lattice sites and the division into the two sets $\Omega_{\mathrm{L}}$ and $\Omega_{\mathrm{P}}$ in Eq. (1) is a dynamic one corresponding to an annealed mixture. Because each site of the lattice is coupled to one of the heat baths, there is no conservation of energy. In the limit where all spins are prone for internal conversions and $T_{1}$ $=T_{2}$, the model reduces to one previously studied by Glotzer and Coniglio [9].

\section{B. Computational details}

The simulations are carried out on a number of different lattice sizes, $N=L \times L, L=32,64,128$, in order to assess finite-size effects. The strength of the drive is fixed to a value of $\Gamma=10^{-3}$. The actual choice of $\Gamma$, which does not influence qualitatively the results to be reported below, has been guided by the results reported previously for the limit $T_{2}$ $\rightarrow \infty[5,6]$. For this value of $\Gamma$, it is possible within reasonable time limits and for the system sizes studied to bring the system to the steady state, and in the case where microstructuring is observed, this structure can convincingly be established within the size of the actual system.

Typical times needed to reach the steady state and to sample the properties of the steady state range from $6 \times 10^{5}$ to $2 \times 10^{6} \mathrm{MCS}$, depending on the values of the temperatures. The steady-state condition is assured by initiating the system in ordered as well as disordered states and by performing increasing as well as decreasing temperature series.

Only a single composition has been investigated, corresponding to a 7:3 mixture and 20\% impurities. Although the populations of the two impurity states fluctuate, they are found effectively to be conserved in the same proportion as that of the major species [5].

In addition to the steady-state average energy $E=\langle H\rangle$, we have calculated the heat capacity functions $C_{1}\left(T_{1}\right)$ $=\left(\partial E / \partial T_{1}\right)_{T_{2}}$ and $C_{2}\left(T_{2}\right)=\left(\partial E / \partial T_{2}\right)_{T_{1}}$, as well as the structure factor to be described in Sec. III B below.

\section{RESULTS}

\section{A. Microstructures and phase diagram}

The phenomenology of the different steady-state phases and possible phase transitions have been explored by systematically varying the two temperatures $T_{1}$ and $T_{2}$ over a wide range of values (in the following given in units of $J / \mathrm{k}_{\mathrm{B}}$ ). The nature of the different types of structure and order of the

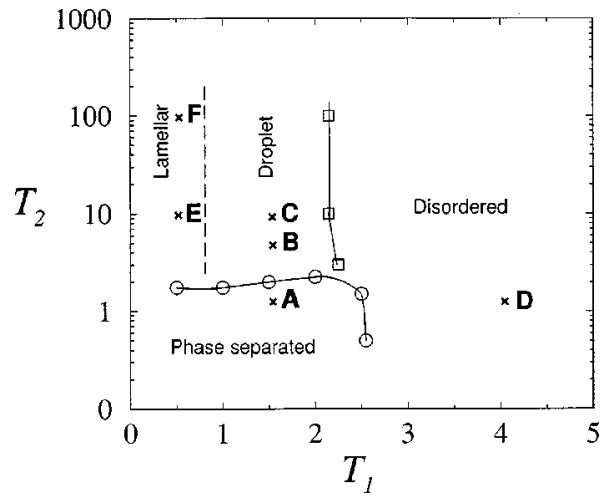

FIG. 2. Steady-state phase diagram for a 7:3 binary lattice-gas model with $20 \%$ active impurities at an activity level of $\Gamma=10^{-3}$. The diagram is spanned by two temperatures, the diffusive temperature $T_{1}$ and the impurity temperature $T_{2}$. The diagram contains three phases, the disordered phase, the ordered (phase-separated) phase, and the microstructured phase, separated by (solid) phase transition lines. The microstructured phase consists of two regions, a droplet region and a lamellar region, separated by a (dashed) transition line. Microconfigurations characteristic of the different phases and regions of the phase diagram at selected points $(X)$, $\mathrm{A}-\mathrm{F}$, are shown in Fig. 1.

phases is inferred by direct inspection of typical printouts of snapshots of microconfigurations. A gallery of the different states found is shown in Fig. 1.

There are basically three types of phases.

(i) An ordered phase of global phase coexistence (A) occurring at low values of the temperatures. In this phase, the two major species are macroscopically phase separated and the impurities dissolved in each of the phases have predominantly the spin value of the mother phase.

(ii) A disordered phase (D) occurring at high values of the temperatures.

(iii) A microstructured phase (similar to that of a binary mixture with microphase separation as in a microemulsion) occurring at high values of the impurity temperature and low values of the diffusion temperature. There are two types of microstructure. A droplet structure (B and C) where the droplet size decreases with increasing values of both temperatures, and a lamellar (or striped) structure ( $E$ and $F$ ) of a characteristic lamellar width. The lamellar phase, which occurs at low values of the diffusion temperature, is induced by the underlying square lattice as discussed below.

The occurrence of the different phases can be described by the steady-state phase diagram that is shown in Fig. 2. The phases are separated by phase lines and steady-state phase transitions that will be discussed below. The regions of stability of the two different types of microstructure are separated by a area of gradual transitions from lamellar microdomains to droplet microdomains.

A quantitative characterization of the structures of the different phases is furnished by the structure factor $S(\vec{q})$ of the minority component $\left(\sigma_{j}=-1\right)$ defined by

$$
S(\vec{q})=\left\langle L^{-2}\left|\sum_{j} \frac{1}{2}\left(1-\sigma_{i}\right) \exp \left(i \vec{r}_{j} \cdot \vec{q}\right)\right|^{2}\right\rangle,
$$

where $\vec{q}=\left(q_{x}, q_{y}\right)$ is a two-dimensional wave vector. The angular brackets denote average over steady-state microcon- 

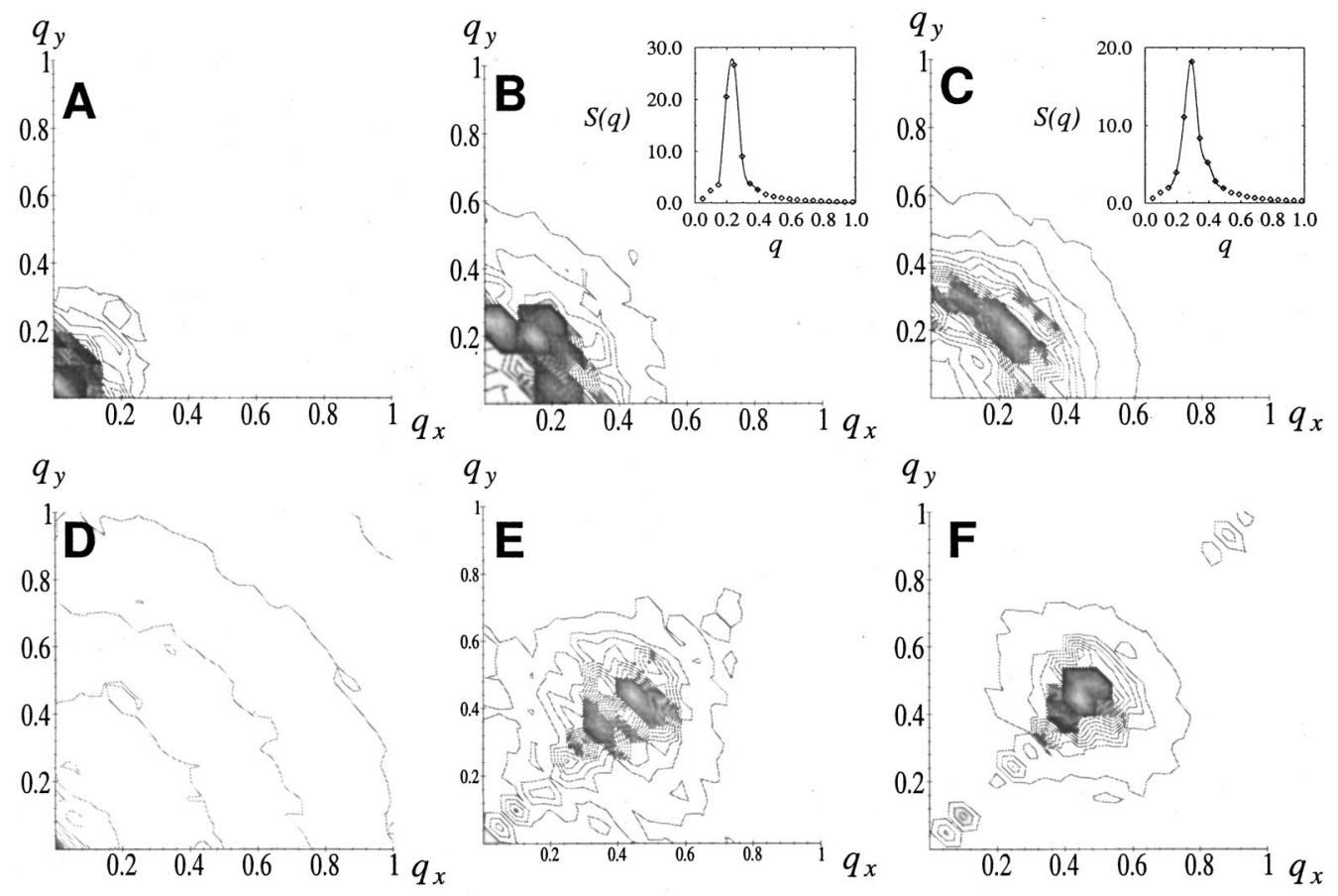

FIG. 3. Contour plots of the structure factor $S\left(q_{x}, q_{y}\right)$, (folded into the first quadrant) for the same six cases, A-F, as shown in Figs. 1 and 2 averaged over 1000 configurations. In order to illustrate the difference in characteristic length scale of the patterns in B and C in Fig. 1 , the insets in B and $\mathrm{C}$ show the circularly averaged structure factors, $S(q)$. The spacing between neighboring contour lines corresponds to changes in the intensity of 1 (A,B,C,E,F) or 0.1 (D).

figurations. For symmetry reasons the structure-factor data are folded into the first quadrant of the wave-vector plane. Contour plots of $S\left(q_{x}, q_{y}\right)$ calculated at the different points $\mathrm{A}-\mathrm{F}$, in the phase diagram in Fig. 2 and corresponding to the characteristic microconfigurations in Fig. 1 are displayed in Fig. 3.

The structure factors for $\mathrm{A}$ and $\mathrm{D}$ in Fig. 3 show the characteristic features of a globally ordered (phaseseparated) phase and a disordered phase, respectively. The structure factors for the phases with a microstructure show distinct features of the nature of the structure and how it varies with the two temperatures.

The droplet structures in Fig. 3, B and C, lead to a diffuse circular band in $S(\vec{q})$ with a width and position of the maximum that depend on the two temperatures. The droplets appear of similar size and with a characteristic spacing. The circularly averaged structure factors $S(q)=S(|\vec{q}|)$, displayed in the insets in $\mathrm{B}$ and $\mathrm{C}$, show that the peak intensity decreases and the position of the peak increases as the value of the impurity temperature $T_{2}$ is increased for fixed value of $T_{1}$. This reflects a decrease in the typical distance between droplets (see Fig. 1) and a wider variation in the distances between the droplets. At the same time, the characteristic droplet size decreases. The same qualitative trends are found for fixed value of $T_{2}$ when the diffusive temperature $T_{1}$ is increased.

The lamellar structures in Fig. 3, E and F, lead to a structure factor with several diffuse peaks along the diagonal corresponding to lamellae along the diagonal of the square lattice. The position of the peak at $\vec{q}=\vec{q}_{0} \simeq\left(\frac{1}{2}, \frac{1}{2}\right)$, which has the largest intensity, determines the lamellar repeat distance, and the peaks at $\vec{q}_{0} / 2$ and $\vec{q}_{0} / 4$ are higher harmonics of the lamellar order. The position of the peak in the right upper corner of frame $\mathrm{F}$ is a measure of the width of the lamellae corresponding to the spin species of the minority component. Under circumstances corresponding to frame E, the variations in the lamellar width are too large to make a clear peak discernible. Taken together with the observation that the peak at $\vec{q}_{0}$ is much more intense in $\mathrm{F}$ than in $\mathrm{E}$, the data therefore show that the lamellae have a more well-defined thickness as well as a more well-defined repeat distance at higher values of $T_{2}$.

The lamellar structures are induced by the square lattice structure. The propagation of the lamellae in the direction along the diagonals of the square lattice is stabilized in the steady state because the barrier for diffusion along the diagonal is smaller than along the axes. This will at low diffusive temperatures single out domain boundaries along the diagonal. At the same time, low temperatures tend to disfavor the curved domain interfaces of droplets.

\section{B. Steady-state phase transitions}

The phase transitions between the different phases have been detected and quantified by systematically varying the two temperatures across the phase diagram. We have found three different steady-state transitions, one from the ordered phase to the disordered phase, one from the ordered phase to the microstructured phase, and one from the microstructured phase to the disordered phase. The transition between the lamellar and droplet regions of the microstructured phase, which has not been studied in detail, is likely not to be a phase transition in a strict sense.

The transitions driven by variation of the diffusive temperature $T_{1}$ are investigated in Fig. 4 for the order-disorder 

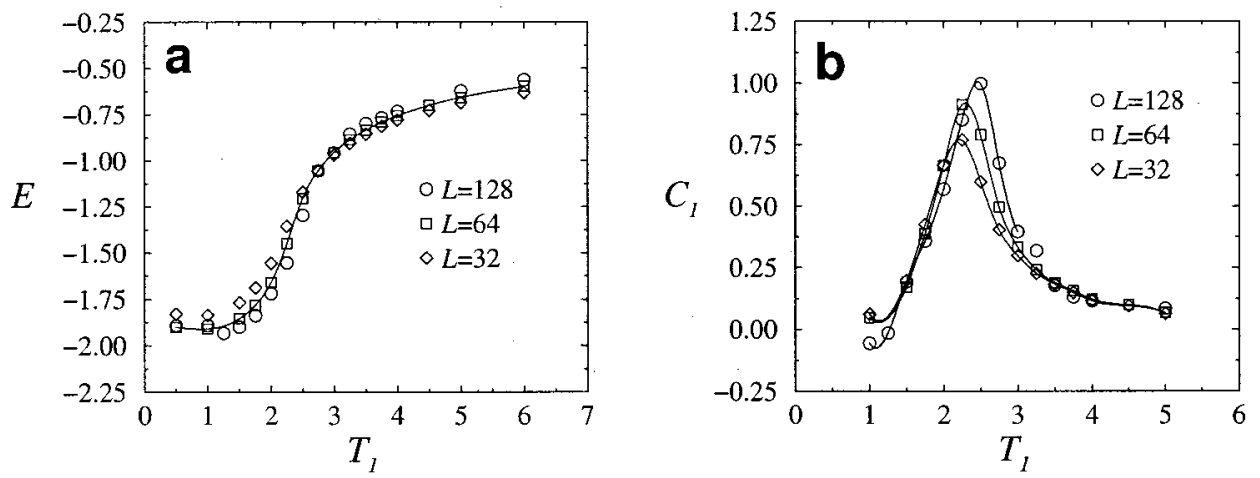

FIG. 4. Internal energy $E$ (in units of $J$ ), and specific heat $C_{1}$ $=\left(\partial E / \partial T_{1}\right)_{T_{2}}$ (in units of $\mathrm{k}_{\mathrm{B}}$ ), as functions of diffusion temperature $T_{1}$. The upper panel, (a) and (b), corresponds to a phase transition from the ordered phase to the disordered phase at an impurity temperature of $T_{2}=1.5$. The lower
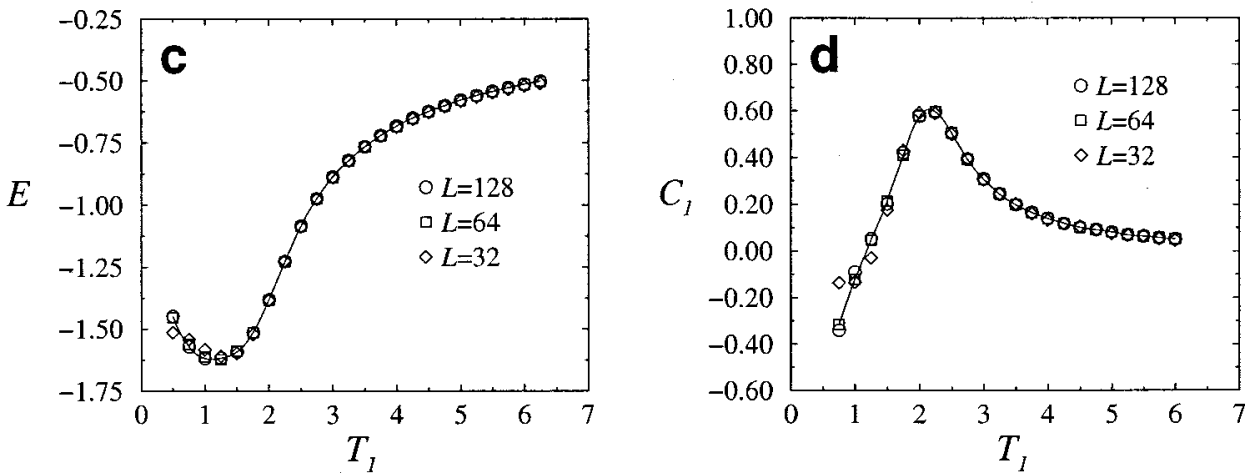
panel, (c) and (d), corresponds to a phase transition from the microstructured phase to the disordered phase at an impurity temperature of $T_{2}=10$. Results are shown for three different system sizes, $L$ $\times L$, as indicated. The solid lines are guides to the eye.

transition [Figs. 4(a) and 4(b)] and for the transition from the microstructured phase to the disordered phase [Figs. 4(c) and 4(d)]. Data are shown for the average internal energy $E$ and the specific heat $C_{1}$.

In the case of the order-disorder transition, Figs. 4(a) and 4(b), the energy is found to display an inflection point corresponding to the peak in the specific heat. The data reveal a systematic finite-size dependence. We estimate the position of the phase transition from the peak in $C_{1}$ for the larger system. In the case of the transition from the microstructured phase to the disordered phase, Figs. 4(c) and 4(d), the size dependence of the data is negligible due to the fact that the characteristic size of the microstructure is much smaller than the system size, and an estimate of the transition region can readily be made from the smooth peak in the specific heat.

From the specific-heat data in Fig. 4, one notes a peculiarity of nonequilibrium thermodynamics in that the specific heat, somewhat counterintuitively, can become negative. The reason for this behavior is that the energy can flow between the two heat baths and an increase in one temperature, i.e., $T_{1}$, can lead to ordering by leakage of energy into the other heat bath, controlled by $T_{2}$. The stronger the nonequilibrium drive is on the system, i.e., the larger the value of the impurity temperature $T_{2}$, the more pronounced this effect can become (as seen on Fig. 4). A related behavior has been found in systems that exhibit "freezing by heating" [28].

The transitions driven by variation of the impurity temperature $T_{2}$, from the ordered phase to the droplet phase are investigated in Fig. 5. Data are shown in Figs. 5(a) and 5(b) for the average internal energy $E$, and the specific heat $C_{2}$, respectively. The internal energy displays a strong size dependence in the ordered phase that quantitatively can be related to the variation of interface-to-area ratio of the minority phase. The location of the transition is approximately determined by the position of the peak of the specific heat. Sup- port for this procedure is provided by the structure-factor plots in Figs. 5(c) and 5(d). The transition occurs at the temperature where the structure factor changes from having a peak at zero wave vector (corresponding to a bulk ferromagnetic phase) to having a peak at finite wave vector corresponding to the droplet structure discussed above.

A couple of remarks are in order concerning the limits where one of the temperatures tends to zero and the model describes equilibrium situations. For $T_{1} \rightarrow 0$, only diffusive motions that cost no energy are allowed. Interparticle exchanges along diagonal domain boundaries involve zero energy and therefore provide a driving force for destabilizing the phase-separated state. The phase-separated state will break up when $T_{2}$ becomes large enough. At the impurity activity level studied, the microstructured phases persist also in the limit $T_{2} \rightarrow \infty$ [5]. For $T_{2} \rightarrow 0$, only impurity conversions with no cost of energy will persist. Such conversions will take place at the domain boundaries of the phaseseparated domains leading to a stabilization of the interface. The order-disorder transition temperature will consequently be larger than that of the corresponding conventional Ising model.

\section{DISCUSSION AND CONCLUSIONS}

We have in this paper presented a simple nonequilibrium statistical mechanical model that illustrates a general principle of pattern formation and small-scale structuring in driven-diffusive systems characterized by two temperatures and no global transport processes. The mobile active impurities in the present model couple to the mobility of domain interfaces in the same way as chemical reactions in binary A-B systems subject to $A \rightleftharpoons B$ conversions $[8,11]$. The nonequilibrium condition in the steady state selects a new length scale that can be tuned to control the microstructuring. In the 

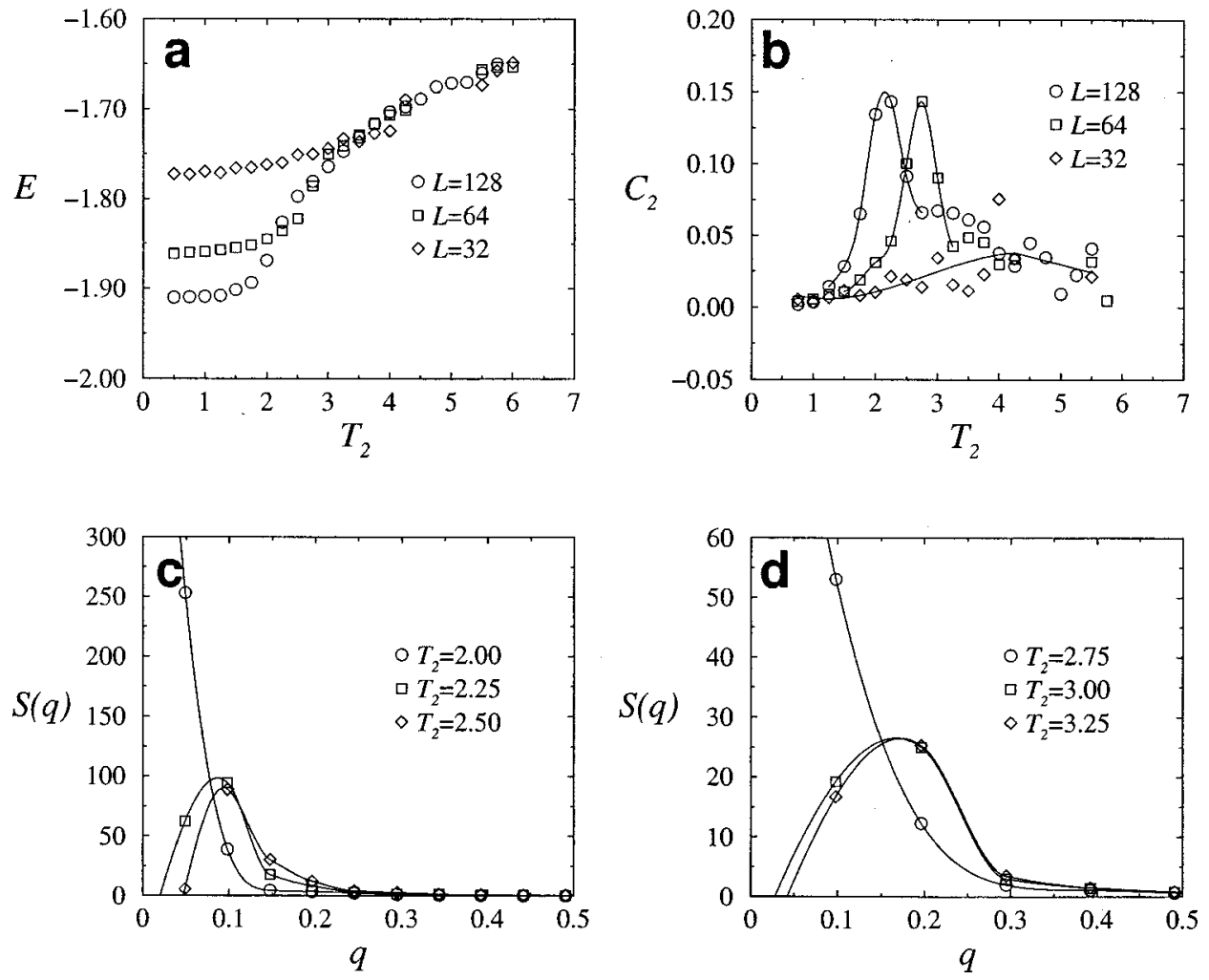

FIG. 5. The upper panel, (a) and (b), shows the internal energy $E$ (in units of $J$ ), and the specific heat $C_{2}=\left(\partial E / \partial T_{2}\right)_{T_{1}}$ (in units of $\mathrm{k}_{\mathrm{B}}$ ), as functions of the impurity temperature $T_{2}$, for phase transitions from the ordered phase to the droplet region of the microstructured phase. In all cases the value of the diffusive temperature is $T_{1}=1.5$. Results are shown for three different system sizes, $L$ $\times L$. The lower panel, (c) and (d), shows the circularly averaged structure factor $S(q)$, for three different impurity temperatures $T_{2}$, spanning the transition from the the ordered phase to the droplet region of the microstructured phase. The data in (c) and (d) are obtained for systems sizes of 128 $\times 128$ and $64 \times 64$, respectively. The solid lines are guides to the eye. present model this leads to stabilization of a droplet phase that under appropriate conditions (cf. the phase diagram in Fig. 2) can be turned into a striped, lamellar phase that is controlled by an instability toward the underlying lattice structure, in the same way as in the Ising lattice model studied by Glotzer et al. [8] but in contrast to the continuum Lennard-Jones model studied by Toxvaerd [11].

The microstructuring in these steady-state systems is different from the microstructuring produced in binary mixtures where a phase-separation process or spinodal-decomposition process has been halted by the presence of a dispersion of surfactants. This is the case in microemulsions [4] that display an equilibrium pattern in the droplet regime that is characterized by a broad and asymmetric size distribution quite different from the more narrow and more symmetric distribution describing patterns such as those shown in Fig. 1. Moreover, these steady-state patterns, in contrast to those due to spinodal-decomposition processes (far away from critical points), are fairly sensitive to thermal fluctuations. It remains unclear in the present case, as in most other nonequilibrium systems, which property the system optimizes in the steady state. It appears from the patterns in Fig. 1 that in the microstructured phases the cost in entropy by formation of well-ordered and packed droplets or lamellae must be compensated by the energy gained by maintaining a certain degree of spin order within the microdomains.

The phase diagram derived in Fig. 2 is expected to be generic for the model studied in this paper. Other choices of values for the model parameters that were not varied in the present context, such as driving strength, $\Gamma$, impurity con- centration, as well as composition of the binary mixture, will not qualitatively change the results.

The dissipation of energy in the model system studied in the present paper takes place under isothermal conditions. This is a realistic condition for, e.g., lipid monolayers on air/water interfaces or lipid-bilayer membranes in water, where the thermocouple between the ultra-thin membrane and the water phase is very fast [29]. It has been proposed that the role of hot spots in these systems could be played by active membrane-bound molecules, such as membrane proteins [6] or photoreactive compounds [30]. Hence the activity of these molecules can control the compartmentalization of the membranes. In the case of transmembrane proteins, whose molecular structure changes during the cycle of the activation, the activity can couple to the local curvature of the membrane in which they are embedded [31]. This leads to the possibility of changing the local membrane bending dynamics and fluctuations by the active proteins and hence to a renormalization of the membrane elastic properties in response to the drive. This phenomenon was recently demonstrated experimentally for a system of lipid bilayers incorporated with the transmembrane protein bacteriorhodopsin [32].

\section{ACKNOWLEDGMENTS}

This work was supported by the Danish Natural Science Council, the Danish Technical Research Council, and the Hasselblad Foundation. O.G.M. acknowledges the Canadian Institute for Advanced Research. Useful correspondence with Royce K. P. Zia is gratefully acknowledged. 
[1] Micelles, Membranes, Microemulsions, and Monolayers, edited by W. M. Gelbart, A. Ben-Shaul, and D. Roux (SpringerVerlag, New York, 1994), p. 608.

[2] M. Laradji, H. Guo, M. Grant, and M. J. Zuckermann, Adv. Chem. Phys. 89, 159 (1995).

[3] L. Leibler, Makromol. Chem., Macromol. Symp. 16, 1 (1988).

[4] M. Laradji, O. G. Mouritsen, S. Toxvaerd, and M. J. Zuckermann, Phys. Rev. E 50, 1243 (1994).

[5] M. C. Sabra, H. Gilhøj, and O. G. Mouritsen, Phys. Rev. E 58, 3547 (1998).

[6] M. C. Sabra and O. G. Mouritsen, Biophys. J. 74, 745 (1998).

[7] B. Schmittmann and R. K. P. Zia, in Phase Transitions and Critical Phenomena, edited by C. Domb and J. L. Lebowitz (Academic Press, London, 1995), Vol. 17, p. 173.

[8] S. C. Glotzer, D. Stauffer, and N. Jan, Phys. Rev. Lett. 72, 4109 (1994).

[9] S. C. Glotzer and A. Coniglio, Phys. Rev. E 50, 4241 (1994).

[10] S. C. Glotzer, E. A. Di Marzio, and M. Muthukumar, Phys. Rev. Lett. 74, 2034 (1995).

[11] S. Toxvaerd, Phys. Rev. E 53, 3710 (1996).

[12] G. H. Fredrickson, Phys. Rev. Lett. 76, 3440 (1996).

[13] B. O'Shaughnessy and U. Sawhney, Phys. Rev. Lett. 76, 3444 (1996).

[14] J. J. Christensen, K. Elder, and H. C. Fogedby, Phys. Rev. E 54, R2212 (1996).

[15] D. Carati and R. Lefever, Phys. Rev. E 56, 3127 (1997).

[16] Q. Tran-Cong and A. Harada, Macromolecules 76, 1162 (1996).
[17] R. Harris and M. Grant, Phys. Rev. B 38, 9323 (1988).

[18] P. L. Garrido, A. Labarta, and J. Marro, J. Stat. Phys. 49, 551 (1987).

[19] J. M. Gonzales-Miranda, P. L. Garrido, J. Marro, and J. L. Lebowitz, Phys. Rev. Lett. 59, 1934 (1987).

[20] J. S. Wang and J. L. Lebowitz, J. Stat. Phys. 51, 893 (1988).

[21] P. L. Garrido, J. Marro, and J. M. Gonzales-Miranda, Phys. Rev. A 40, 5802 (1989).

[22] H. W. J. Blöte, J. R. Heringa, A. Hoogland, and R. K. P. Zia, J. Phys. A 23, 3799 (1990).

[23] Z. Cheng, P. L. Garrido, J. L. Lebowitz, and J. L. Vallés, Europhys. Lett. 14, 507 (1991).

[24] E. L. Praestgaard, H. Larsen, and R. K. P. Zia, Europhys. Lett. 25, 447 (1994).

[25] K. E. Bassleer and Z. Rácz, Phys. Rev. Lett. 73, 1320 (1994); Phys. Rev. E 52, R9 (1995).

[26] A. Szolnoki, G. Szabo, and O. G. Mouritsen, Phys. Rev. E 55, 2255 (1997).

[27] A. Szolnoki, Phys. Rev. E 60, 2425 (1999).

[28] D. Helbing, I. J. Farkas, and T. Vicsek, Phys. Rev. Lett. 84, 1240 (2000).

[29] M. Bloom, E. Evans, and O. G. Mouritsen, Q. Rev. Biophys. 24, 293 (1991).

[30] M. Tanaka and Y. Yonezawa, J. Phys. Chem. 1000, 5160 (1996).

[31] J. Prost and R. Bruinsma, Europhys. Lett. 33, 321 (1996).

[32] J.-B. Manneville, P. Bassereau, D. Lévy, and J. Prost, Phys. Rev. Lett. 82, 4356 (1999). 